\title{
BMJ
}

\section{Effect of repeated invitations on uptake of colorectal cancer screening using faecal occult blood testing: analysis of prevalence and incidence screening}

\author{
R J C Steele, professor of surgery, ${ }^{1,2}$ I Kostourou, research fellow, ${ }^{1,2}$ P McClements, statistician, ${ }^{3}$ C Watling, \\ statistician, ${ }^{3} \mathrm{G}$ Libby, research statistician, ${ }^{2} \mathrm{D}$ Weller, James Mackenzie professor of general practice, ${ }^{4} \mathrm{D} \mathrm{H}$ \\ Brewster, director, Scottish cancer registry, ${ }^{3}$ R Black, head of programme, ${ }^{3} \mathrm{~F}$ A Carey, professor of pathology, ${ }^{5}$ \\ C Fraser, director of screening laboratory ${ }^{2}$
}

${ }^{1}$ Department of Surgery, University of Dundee, UK

${ }^{2}$ Scottish Bowel Screening Centre, King's Cross Hospital, Dundee

IInformation Services, National Services Scotland, UK

${ }^{4}$ Centre for Population Health Sciences, University of Edinburgh, UK

${ }^{5}$ Department of Pathology, University of Dundee

Correspondence to: RJ C Steele, Department of Surgery and Molecular Oncology, Ninewells Hospital and Medical School, Dundee DD1 9SY, UK r.j.c.steele@dundee.ac.uk

Cite this as: BMJ 2010;341:C5531 doi:10.1136/bmi.c5531

\section{ABSTRACT}

Objective To analyse the effects of prevalence and incidence screening on uptake and detection of cancer in an ongoing, dynamic programme for colorectal screening using faecal occult blood testing.

Design Analysis of prevalence and incidence screening.

Setting Three rounds of biennial colorectal screening using the guaiac faecal occult blood test in east and north east Scotland, March 2000 to May 2007.

Participants Adults aged 50-69.

Main outcome measures Uptake of screening, test positivity (percentage of those invited who returned a test that was positive and triggered an invitation for colonoscopy), positive predictive value, and stage of cancer.

Results of 510990 screening episodes in all three rounds, 248998 (48.7\%) were for prevalence, 163483 (32.0\%) were for first incidence, and 98509 (19.3\%) were for second incidence. Uptake of a first invitation for prevalence screening was $53 \%$ and for a second and third invitation was $15 \%$ and $12 \%$. In the cohort invited for the first round, uptake of prevalence screening rose from $55 \%$ in the first round to $63 \%$ in the third. The uptake of first incidence screening on a first invitation was $54 \%$ and on a second invitation was $86 \%$ and on a first invitation for second incidence screening was $46 \%$. The positivity rate in prevalence screening was $1.9 \%$ and the uptake of colonoscopy was $87 \%$. The corresponding values for a first incidence screen were $1.7 \%$ and $90 \%$ and for a second incidence screen were $1.1 \%$ and $94.5 \%$. The positive predictive value of a positive faecal occult blood test result for cancer was $11.0 \%$ for prevalence screening, $6.5 \%$ for the first incidence screen, and $7.5 \%$ for the second incidence screen. The corresponding values for the positive predictive value for adenoma were $35.5 \%$, $29.4 \%$, and $26.7 \%$. The proportion of cancers at stage I dropped from $46.5 \%$ for prevalence screening to $41 \%$ for first incidence screening and 35\% for second incidence screening.

Conclusions Repeat invitations to those who do not take up the offer of screening increases the number of those who accept, for both prevalence screening and incidence screening. Although the positive predictive value for both cancer and adenomas fell between the prevalence screen and the first incidence screen, they did not fall between the first and second incidence screens. The deterioration in cancer stage from prevalence to incidence screening suggests that some cancers picked up at incidence screening may have been missed on prevalence screening, but the stage distribution is still favourable. These data vindicate the policies of continuing to offer screening to those who fail to participate and continuing to offer biennial screening to those who have accepted previous offers.

\section{INTRODUCTION}

In screening programmes where the screening test is offered at regular intervals, the terms prevalence and incidence screening are widely used. Prevalence screening refers to the first time a population is screened and the term incidence screening is used to describe subsequent screens. ${ }^{1}$ Strictly speaking this nomenclature is incorrect, as no screening test is sufficiently sensitive to detect all prevalent disease in a population, and disease detected at incidence screening may have been present at the time of prevalence screening but was missed by the test. Nevertheless, study of these distinct screening categories is important as it provides information that has central implications for the performance of a programme and in particular permits estimation of the value of repeated invitations to participate. In screening for colorectal cancer comparatively little is known about the relative effects of prevalence and incidence screening, as the only information that is available from long term screening programmes comes from population based trials of screening using the guaiac faecal occult blood test, ${ }^{2-7}$ where the emphasis was on the effect of the programmes on disease specific mortality.

As a result of these trials the UK departments of health commissioned a demonstration pilot to test the feasibility of introducing a screening programme using 
the guaiac faecal occult blood test into the National Health Service. ${ }^{8}$ The pilot was carried out in two areas, one in England and one in Scotland. ${ }^{9}$ In the Scottish arm of this pilot, three biennial rounds of screening were carried out between 2000 and $2006 .{ }^{10} \mathrm{We}$ examined the differential effect of prevalence and incidence screening in the Scottish arm of the pilot.

\section{METHODS}

The Scottish arm of the UK demonstration pilot was carried out in Grampian, Tayside, and Fife, using biennial guaiac faecal occult blood testing (Haemascreen; Immunostics, Ocean, NJ). Participants with five to six positive windows on the initial test (strong positive) were offered colonoscopy, but if one to four windows were positive (weak positive) participants were asked to complete a further guaiac faecal occult blood test, and if subsequent windows were positive colonoscopy was offered. Minor variations occurred in this algorithm between the three rounds and these are detailed elsewhere. ${ }^{10}$

Men and women aged 50 to 69 and living in the three pilot NHS boards were invited for screening. They were identified by the community health index and sent a test kit and an invitation to participate by post from a single screening centre located in Dundee. The community health index is a unique identifying number for everyone registered with a general practitioner in Scotland. The number is made up of the date of birth followed by four digits from which sex can be identified; the precise accuracy of the community health index in identifying place of residence is unknown, but fewer than $3 \%$ of the invitations were returned undelivered to the screening centre. Completed tests were sent back in specially designed foil envelopes to the unit laboratory for analysis. A specialist nurse contacted those with a positive test result and organised

Screening episode categories occurring in first three rounds of screening programme First invitation prevalence-invitees responding to their first invitation to be screened for the first time

Second invitation prevalence-invitees responding to their second invitation to be screened for the first time

Third invitation prevalence-invitees responding to their third invitation to be screened for the first time

First invitation first incidence-invitees responding to their first invitation to be screened for the second time

Second invitation first incidence-invitees responding to their second invitation to be screened for the second time- that is, having completed a prevalence screen, they did not respond to the next invitation but did to the subsequent invitation

First invitation second incidence-invitees responding to their first invitation to be screened for the third time

Possible categories of screening episodes in the first three rounds:

First round-first invitation prevalence

Second round-first invitation prevalence, second invitation prevalence, and first invitation first incidence

Third round - any of the six categories above colonoscopy after informed consent had been obtained.

Data collectors employed by the screening unit collected information for key performance indicators. These were analysed by Information Services Scotland, a division of NHS National Services Scotland. All analyses were carried out on anonymised data.

Unlike randomised trials that limited their analyses to participants recruited in the first round, the second and third rounds of the demonstration pilot screened people who were being invited to prevalence screening for the first time. In addition, in every round of a screening programme (other than the first) there is potential to carry out prevalence screening in people who failed to respond to a previous invitation. Similarly, it is possible to carry out incidence screening for the first time in those who had failed to respond to a previous invitation, and this also holds for second and subsequent incidence screens. To add further complexity, it is possible for people to miss incidence screens but to respond to subsequent invitations - for example, someone who undergoes prevalence screening at the first invitation (in the first screening round) may decline a first incidence screen in the second round but accept it when it is offered again in the third round.

Thus in the first round of the demonstration pilot, all screening episodes were by necessity prevalence screens from a first invitation. In the second round there was a mixture of prevalence screens from first and second invitations and first incidence screens from a first invitation. By the third round, there was a complex mix of prevalence screens from first, second, and third invitations; first incidence screens from first and second invitations; and second incidence screens from a first invitation (box).

To assess the effect of prevalence and incidence screening and hence to gauge the value of continuing to issue invitations for screening regardless of whether individuals responded to the invitation, we calculated the uptake of the different invitations for prevalence and incidence screening. In addition, for prevalence, first incidence, and second incidence screening we determined the uptake of colonoscopy, test positivity, positive predictive value of the test, and stage of screen detected cancer.

\section{RESULTS}

In all three rounds combined, prevalence screens totalled 248998 (169508 screens in the first round, 38283 in the second, 41207 in the third) out of 624421 invitations. Of these invitations, 409559 were first invitations, 136503 second invitations, and 78359 third invitations.

Similarly, incidence screens totalled $261992(126618$ in the second round, 135374 in the third) of 645362 invitations. Of these invitations, 307235 were first incidence screens on a first invitation, 123142 were first incidence screens on a second invitation, and 214985 were second incidence screens on a first invitation. 


\section{Uptake}

Uptake of the first invitation to prevalence screening was $53 \%$, and this dropped from $55 \%$ in the first round to $45 \%$ in the second and $48 \%$ in the third (fig 1); the uptake of the second invitation for prevalence screening was $15 \%$, and this dropped further to $12 \%$ for the third invitation (fig 1). Of the cohort invited to prevalence screening in the first round, however, uptake of prevalence screening had risen to $63 \%$ by the third round (fig 1 ).

The uptake of the first incidence screening on a first invitation was $54 \%$, first incidence screening on the second invited was $86 \%$, and second incidence screening on a first invitation was $46 \%$ (fig 1).

\section{Uptake of colonoscopy}

Uptake of colonoscopy in those with a positive guaiac faecal occult blood test result was $87 \%$ for prevalence screening. This rose steadily to $90 \%$ for first incidence screening and to $94.5 \%$ for second incidence screening (fig 2).

\section{Positivity}

The test positivity (proportion of returned guaiac faecal occult blood test results that were positive for blood) was $1.9 \%$ for prevalence screening, which fell to $1.7 \%$ for first incidence screening and to $1.1 \%$ for second incidence screening (fig 2).

\section{Positive predictive value for cancer and adenoma}

The positive predictive value for cancer was $11.0 \%$ for prevalence screening, which fell to $6.5 \%$ for first
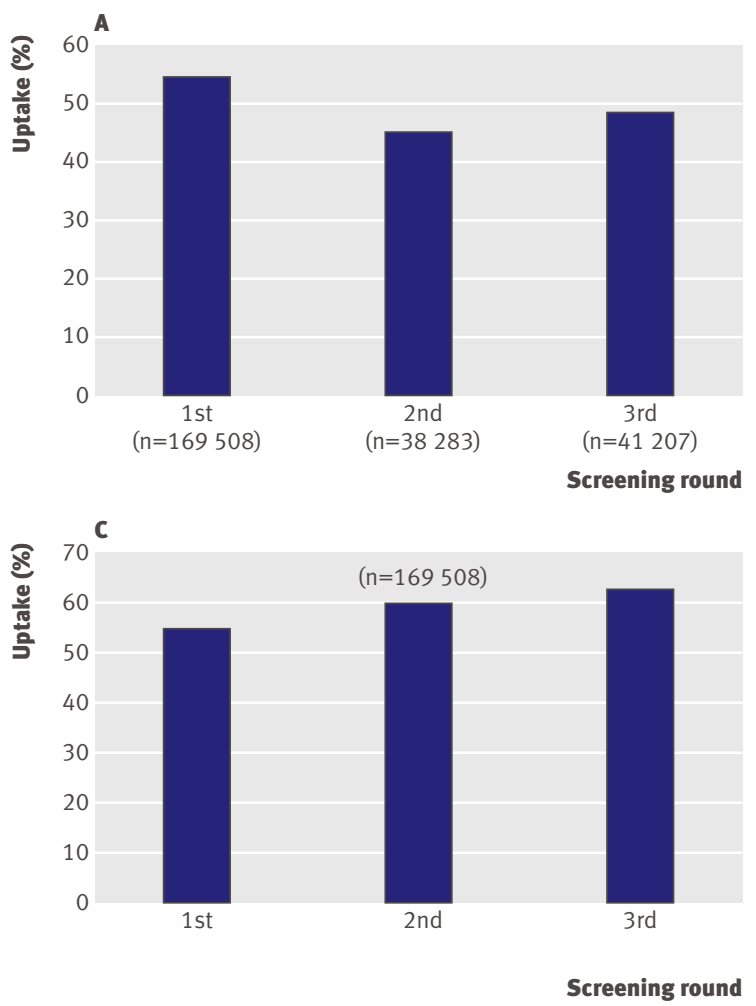

incidence screening but rose slightly to $7.5 \%$ for second incidence screening (fig 2). For adenoma the corresponding positive predictive values were $35.5 \%$, $29.4 \%$, and $26.7 \%$, respectively (fig 2 ).

\section{Stage at diagnosis}

In prevalence screening the proportion of screen detected cancers diagnosed at stage I (Dukes' A) was $46.5 \%$, but this fell to $41 \%$ for first incidence screening and to $35 \%$ for second incidence screening. However, it was also found that $6 \%$ of cancers diagnosed by prevalence screening were stage IV (Dukes' "D"), but this fell to $2 \%$ for first incidence screening, and by second incidence screening no cancer diagnosed was stage IV (fig 3).

\section{DISCUSSION}

This study clearly shows that repeated invitations to screening using faecal occult blood testing have an effect on uptake. Previously, however, relatively little was known about the true effect of prevalence and incidence screening. Even in breast cancer screening, studies that examine prevalence and incidence focus on screening rounds despite the fact that, with the exception of the first round, all rounds are a mixture of prevalence and incidence screening. ${ }^{11}{ }^{12}$ In the UK breast screening programme, however, it is known that cancer detection rates are lower in incidence screens than in prevalence screens. ${ }^{13}$

In colorectal cancer some information can be gleaned from the previous population based trials.
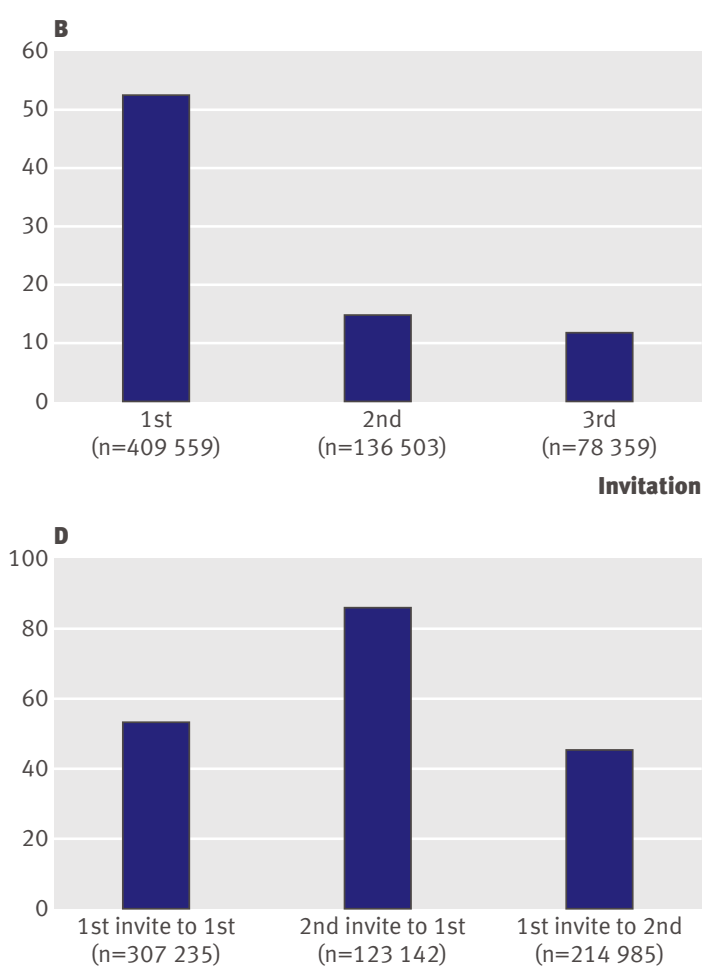

Invitation to incidence screening

Fig 1| (A) Uptake of prevalence screening on first invitation by round, (B) uptake of prevalence screening by invitation, (C) cumulative uptake of prevalence screening in those invited in first round over all three rounds, and (D) uptake of first and second incidence screening by invitation 



Fig 2 (A) Uptake of colonoscopy in those with a positive screening test, (B) test positivity (defined as percentage of those invited who returned a test that was definitively positive and triggered an invitation for colonoscopy), and positive predictive value of the guaiac faecal occult blood test for cancer (C) and for adenoma (D)

Reports from a study in Nottingham ${ }^{3}$ do not allow analysis of uptake by prevalence or incidence screening, but information is available on positivity, positive predictive value, and stage at diagnosis. In a Danish study, ${ }^{4}$ those who declined an invitation to be screened were not invited again and hence this study does not permit detailed scrutiny of the effect of repeated invitations to screening. A Swedish study ${ }^{5}$ reported on prevalence screening, first rescreening, and second rescreening but only in terms of the three screening rounds; it did not take into account the mixture of prevalence and incidence screening in the second two rounds and it did not report on the effect of repeated invitations to prevalence screening. Similarly a French study, ${ }^{6}$ in which small geographical areas were allocated to either screening using guaiac faecal occult blood testing or no screening, reported its results in terms of screening round rather than by incidence or prevalence.

Thus to our knowledge our report is the first to separate out prevalence and incidence screening by individual. This approach is important as it is the only way of accurately estimating the effect and hence the utility of repeated invitations for both prevalence and incidence screening.

In the Scottish demonstration pilot the uptake of prevalence screening was much higher for first invitations than for subsequent invitations, although over three rounds repeated invitations increased overall uptake of prevalence screening by $8 \%$. This may seem modest, but it represents 13561 people who would not have been screened had a decision been taken not to reinvite those who did not respond to an invitation, as was done in the Danish trial. ${ }^{4}$ For the rolled-out programmes across the United Kingdom, and in all countries with screening programmes that rely on repeated invitations, ${ }^{14}$ this has substantial implications. The drop in uptake of prevalence screening on a first invitation observed between the first and subsequent rounds is probably a function of age, as prevalence screening in the first round embraced the whole of the 50-69 age range, unlike the second and third rounds, and it is known that uptake increases with increasing age. ${ }^{15}$

The uptakes of the first invitation to first and second incidence screening were similar to the uptake of the first invitation to prevalence screening, which is lower than might be hoped as these were people who had



Fig 3 | Stage of screen detected cancer at diagnosis. stage I (Dukes' A), stage II (Dukes' B), stage III (Dukes' C), stage IV (Dukes' "D") 
already engaged in the screening process. However, the uptake of the second invitation to first incidence screening was considerably higher than the first invitation to incidence screening, indicating that those who decline an offer of incidence screening are likely to accept further invitations. It is therefore worth while continuing to offer incidence screening to those who have defaulted in the past.

The uptake of colonoscopy in those who had a positive guaiac faecal occult blood test result rose by about $8 \%$ between prevalence screening and second incidence screening. This presumably reflects the fact that those who undergo second incidence screening represent a particularly compliant group.

The drop in test positivity between prevalence screening and second incidence screening is not particularly surprising as the burden of neoplastic disease in people undergoing incidence screening should be less, owing to disease detection in previous screens. This is in keeping with the Nottingham study, which found a positivity of $2.1 \%$ at the first invitation, $1.2 \%$ for rescreening within 27 months, and $1.9 \%$ with rescreening after 27 months. $^{3}$

The positive predictive value for cancer also followed the expected pattern of a drop from prevalence screening to first incidence screening. This is explained by a reduction in cancer prevalence brought about by the prevalence screen but without a parallel reduction in false positive test results. Interestingly, the positive predictive value did not drop between the first and second incidence screens, suggesting that a steady state may be reached after the prevalence screen. A similar pattern was seen for positive predictive value for adenoma, although this did seem to drop between first and second incidence screens. It is surprising therefore that previous studies have not observed these trends. The positive predictive value for cancer rose between first screening and rescreening in the Nottingham study ${ }^{3}$ and significant decreases in the positive predictive value were not seen between the first and subsequent rounds in the Danish study ${ }^{4}$ or the Swedish study. ${ }^{5}$ In the French study ${ }^{6}$ the positive predictive value for cancer fell from the first to the second screening round and then rose to a level that was consistently higher than the first round in the subsequent four rounds. The explanation for the discrepancy between the present study and previous studies may be related to the mixture of prevalence and incidence screening in the various rounds, but this does not explain the findings in the Nottingham study.

The data on stage at diagnosis revealed an interesting pattern. The proportion of people with early (stage I) cancer fell progressively from prevalence screening to first incidence screening. However, the proportion of people with advanced (stage IV) cancer also fell. This indicates that the pool of people with unrecognised metastatic disease is reduced by the prevalence screen, but this does not hold for the more advanced stages of localised disease.

Various conclusions can be made from these results. Firstly, it is clear that repeated invitation for prevalence screening increases the total number of people who undergo screening at least once. It also indicates that repeat invitations for incidence screening have a similar effect. Even more fundamental is the finding that incidence screening continues to detect important neoplastic disease. Thus the assumption that repeated screens are potentially beneficial seems to be vindicated. It could be argued, however, that the resource required for repeat invitations might be better used in other ways - for example, to encourage those with a positive test result to follow through with colonoscopy. As yet, however, an evidence base to indicate an appropriate intervention that would be effective in this respect is lacking.

The patterns of stage at diagnosis for screen detected cancer raise some particularly interesting issues. It might have been expected that the prevalence screen would have detected those people in the population harbouring advanced (but as yet asymptomatic) disease in addition to those with early disease, and that subsequent screens would detect an increasing proportion of early disease. Whereas this assumption seems to be correct in terms of patients with metastatic disease, in those with localised disease the proportion with early cancer actually drops with progressive screening. This suggests that at least a proportion of cancers diagnosed on incidence screening are not truly incident cancers but cancers that were missed on previous screens; this is not surprising given the interval cancer rate of around $30 \%$ that has been previously reported. ${ }^{10}$

The results of this study have important implications for population based screening programmes that test faeces for blood. They show for the first time that repeated invitations to both prevalence screening and incidence screening do pay dividends in terms of increasing uptake, although this is more pronounced with incidence screening (that is, in those who have already engaged with the process). In addition, we have found that both first and second incidence screening are associated with significant yields of neoplasia and that the positive predictive value for cancer does not drop between first and second incidence screening.

However, the deterioration of stage distribution of operable screen detected cancers strongly suggests that at least some cancers detected by incidence screening had been missed by previous screens. This highlights the importance of improving the screening test, and currently the two strongest candidates are flexible sigmoidoscopy and faecal immunological testing, which, unlike the guaiac faecal occult blood test, is specific for human haemoglobin. A randomised trial showed faecal immunological testing to be more sensitive for cancer and adenomas and more acceptable than the guaiac faecal occult blood test. ${ }^{16}$ With the advent of quantitative faecal immunological testing, where the positivity threshold can be set to take account of colonoscopy capacity, screening using faecal immunological testing is now a feasible option.

A recently published randomised trial of single flexible sigmoidoscopy showed significant reductions in cumulative colorectal cancer mortality and incidence ${ }^{17}$ 


\section{WHAT IS ALREADY KNOWN ON THIS TOPIC}

In biennial screening for colorectal cancer using guaiac faecal occult blood testing (gFOBT) the test positivity rate and the positive predictive value drops between the first round and subsequent rounds

The differential effect of prevalence and incidence screening on these variables is unknown

The uptake of prevalence and incidence screening has not been studied in this context, so that the effect of repeated invitations to be screened is unknown

\section{WHAT THIS STUDY ADDS}

Uptake of prevalence screening is greatest at the first invitation, but repeat invitations increase uptake by about $8 \%$ over three rounds

Incidence screening detects significant neoplastic disease, and although the positive predictive value of first incidence screening is less that prevalence screening, it does not drop with second incidence screening

The stage at diagnosis of operable screen detected cancer deteriorates between prevalence and first incidence screens, suggesting that incidence screening with gFOBT is detecting tumours that have been missed on previous screens and presents an attractive alternative to screening using a guaiac faecal occult blood test or faecal immunological test; good evidence suggests that single flexible sigmoidoscopy is more sensitive for cancer and adenomas than either a single screening using a guaiac faecal occult blood test or faecal immunological test. ${ }^{17}$ However, the flexible sigmoidoscopy trial was effectively carried out in a volunteer cohort and it is not clear what the population uptake would be in the United Kingdom or elsewhere. Independent evidence from the Netherlands indicates that uptake of flexible sigmoidoscopy ${ }^{18}$ is significantly less than that for faecal testing and that those who are invited for screening find flexible sigmoidoscopy more burdensome. ${ }^{19}$ In addition, although a single test may have advantages, without repeat invitations an opportunity for increasing uptake is lost.

The future direction of population screening for colorectal cancer screening is uncertain, and new more sensitive and specific tests may become available. In the meantime, however, it seems that both faecal immunological testing and flexible sigmoidoscopy have advantages over guaiac faecal occult blood testing, and indeed faecal immunological testing and flexible sigmoidoscopy may be used effectively in concert. This study has shown the importance of repeated invitations and highlights the need for careful monitoring of the effects of prevalence and incidence screening.

Contributors: RJCS, principal investigator, prepared the manuscript. IK prepared the manuscript and designed the tables. PMcC, $\mathrm{CW}$, and $\mathrm{GL}$ provided and analysed the data. DW, DHB, and RB contributed to the study design and manuscript. FAC provided the pathology data. CF provided the faecal occult blood test results and contributed to the manuscript. RJCS and CF are guarantors.

Funding: The pilot was funded by the Scottish Government Health Department and the analysis was supported by a grant from the Chief Scientist Office (grant No CZH/6/4), Scottish Government Health Department to establish a bowel screening research unit. The University of Dundee acts as the sponsor, and administers the grant that supports the bowel screening research unit. All authors are independent from the funders in terms of freedom to publish.

Competing interests: All authors have completed the Unified Competing Interest form at www.icmje.org/coi_disclosure.pdf (available on request from the corresponding author) and declare: no support from any company for the submitted work; no financial relationships with any companies that might have an interest in the submitted work in the previous 3 years; no other relationships or activities that could appear to have influenced the submitted work.

Ethical approval: Ethical approval was not sought for the demonstration pilot. This was a decision made by the National Screening Committee and endorsed by the UK Departments of Health on the grounds that screening for colorectal cancer using faecal occult blood is of proved efficacy, and the study constituted evaluation of the feasibility of introducing a screening programme into the NHS. Permission to access and analyse the anonymised data presented in this paper was granted by the Community Health Index Advisory Board and the Privacy Advisory Committee, National Services Scotland.

Data sharing: No additional data available.

1 Duffy SW, Gabe R. What should the detection rates of cancers be in breast screening programmes? Br J Cancer 2005;92:597-600.

2 Mandel JS, Bond JH, Church JR, Snover DC, Bradley GM, Schuman LM, et al. Reducing mortality from colorectal cancer by screening for faecal occult blood. N Engl J Med 1993;328;1365-71.

3 Hardcastle JD, Chamberlain JO, Robinson MHE, Moss SM, Amar SS, Balfour TW, et al. Randomised controlled trial of faecal occult blood screening for colorectal cancer. Lancet 1996;348;1472-7.

4 Kronborg O, Fenger C, Olsen J, Jorgensen OD, Sondergaard O. Randomised study of screening for colorectal cancer with faecal occult blood test. Lancet 1996;348;1467-71.

5 Lindholm E, Brevinge $\mathrm{H}$, Haglind $\mathrm{E}$. Survival benefit in a randomized clinical trial of faecal occult blood screening for colorectal cancer. $\mathrm{Br}$ Surg 2008;95:1029-36.

6 Faivre J, Dancourt V, Lejeune C, Tazi MA, Lamour J, Gerard D, et al. Reduction in colorectal cancer mortality by faecal occult blood screening in a French controlled study. Gastroenterology 2004:126:1674-80.

7 Hewitson P, Glasziou P, Watson E, Towler B, Irwig L. Cochrane systematic review of colorectal cancer screening using the faecal occult blood test (hemoccult): an update. Am J Gastroenterol 2008;103:1541-9.

8 Steele RJC, Parker R, Patnick J, Warner J, Fraser C, Mowat NA, et al. A demonstration pilot for colorectal cancer in the UK: a new concept in the introduction of health care strategies. J Med Screen 2001;8:197-202.

9 UK Colorectal Cancer Screening Pilot Group. Results of the first round of a demonstration pilot of screening for colorectal cancer in the United Kingdom. BMJ 2004;329:133-5.

10 Steele RJC, McClements PL, Libby G, Black R, Morton C, Birrell J, et al. Results from the first three rounds of the Scottish demonstration pilot of FOBT screening for colorectal cancer. Gut 2009;58:530-5.

11 Anttila A, Koskela J, Hakama M. Programme sensitivity and effectiveness of mammography service screening in Helsinki, Finland. I Med Screen 2002;9:153-8.

12 Otten JD, van Dijick JA, Peer PG, Straatman H, Verbeek AL, Mravunac M, et al. Long term breast cancer screening in Nijmegen, The Netherlands: the nine rounds from 1975-92. J Epidemiol Community Health 1996;50:353-8.

13 Blanks RG, Moss SM, Patnick J. Results from the UK NHS breast screening programme 1994-1999. I Med Screen 2000;7:195-8.

14 Benson VS, Patnick J, Davies AK, Nadel MR, Smith RA, Atkin WS. Colorectal cancer screening: a comparison of 35 initiatives in 17 countries. Int J Cancer 2008;122:1357-67.

15 Steele RJ, Kostourou I, McClements P, Watling C, Libby G, Weller D, et al. Effect of gender, age and deprivation on key performance indicators in a FOBT based colorectal screening programme. J Med Screen 2010;17:68-74.

16 Van Rossum LG, van Rijn AF, Laheij RJ, van Oijen MG, Fockens P, van Krieken $\mathrm{HH}$, et al. Random comparison of guaiac and immunochemical fecal occult blood tests for colorectal cancer in a screening population. Gastroenterology 2008;135:82-90.

17 Atkin WS, Edwards R, Kralj-Hans I, Wooldrage K, Hart AR, Northover JM, et al. Once-only flexible sigmoidoscopy screening in prevention of colorectal cancer: a multicentre randomised controlled trial. Lancet 2010;375:1624-33.

18 Hol L, van Leerdam ME, van Ballegooijen M, van Vuuren AJ, van Dekken H, Reijerink JC, et al. Screening for colorectal cancer: a randomised trial comparing guaiac-based and immunochemical faecal occult blood testing and flexible sigmoidoscopy. Gut 2010;59:62-8

19 Hol L, de Jonge V, van Leerdam ME, van Ballegooijen M, Looman CW, van Vuuren Al, et al. Screening for colorectal cancer: comparison of perceived test burden of guaiac-based faecal occult blood test, faecal immunochemical test and flexible sigmoidoscopy. Eur Cancer 2010;46:2059-66.

Accepted: 24 August 2010 